\title{
Evidenzbasierte Konzepte der motorischen Frührehabilitation
}

Neurol Rehabil 2016; 22(3): 209-216

(C) Hippocampus Verlag 2016

DOI 10.14624/NR150816.004

\author{
S. Thomas' ${ }^{1}$ M. Pohl², J. Mehrholz'1,3
}

\section{Zusammenfassung}

Frühe Rehabilitation von Patienten nach Hirnschädigung ist wesentlich, um Funktionen, Aktivitäten und Teilhabe am Leben wieder zu ermöglichen. Jedoch sind für den Erfolg der Frührehabilitation die Dosis der Intervention und die Schwere der neurologischen Schädigung zu berücksichtigen. Frührehabilitation bei schweren Hirnverletzungen sollte frühzeitig sowie mit angepasster Dosis und bei relativ und entsprechender niedriger Intensität in den ersten 2 Wochen durchgeführt werden. Patienten mit leichter Schädigung können bereits von einem intensiven Training innerhalb der ersten Woche profitieren. Spätestens 2 Wochen nach dem Akutereignis ist ein hoch intensives Training aller Patienten mit Hirnschädigung effektiv möglich. Bei Patienten mit auf Intensivstation erworbenem Schwächesyndrom und schweren peripheren Paresen kann die Mobilisation innerhalb der ersten 48 Stunden sicher durchgeführt werden. Ein tägliches intensives Training in den ersten 4 Wochen innerhalb der Frührehabilitation kann die Mobilität dieser Patienten ebenfalls verbessern. Aber nicht nur der Zeitpunkt der Frührehabilitation, sondern auch die Inhalte der Therapien spielen eine wesentliche Rolle. Bei leichten Armparesen nach Schlaganfall sollte z. B. die Constrained Induced Movement Therapy bevorzugt werden. Hingegen sind bei schweren Paresen uni- sowie auch bilaterale repetitive Trainingsmaßnahmen effektiv. Hier kann zum Beispiel sehr gut das roboterassistierte Armtraining angewandt werden. Patienten, die nach einem Schlaganfall nicht mehr gehen können, profitieren deutlich von einem frühen Gehtraining mit Hilfe der elektromechanisch assistierten Gangtherapie. Schlaganfallpatienten hingegen, die bereits gehen können ( $F A C \geq 3$ ), profitieren vor allem von einem sehr intensiven Training wie einem Sprinttraining auf dem Laufband bezüglich Gehausdauer, Gehfähigkeit und Gehgeschwindigkeit. Bei Patienten mit prolongiertem Weaning und erworbenem Schwächesyndrom auf der Intensivstation sind ebenfalls aktive Maßnahmen der therapeutische Hauptschwerpunkt. Vor allem die allgemeine Mobilisierung in den Sitz, das Training des selbstständigen Aufstehens, des Stehens und Gehens stehen im Vordergrund. Maßnahmen zur Stärkung der inspiratorischen Atemmuskulatur und zur Verbesserung der Muskelkraft sowie ein Ausdauertraining können zur Verbesserung der Mobilität beitragen.

Schlüsselwörter: Frührehabilitation, Armfunktionen, Gehfähigkeit

\section{Einleitung}

Frühe motorische Rehabilitation in der Neurologie: Wann mobilisieren und warum?

Die motorische Rehabilitation von Patienten nach Hirnschädigung ist bedeutend, um eine Wiederherstellung von Funktionen, Aktivitäten und Teilhabe am Leben zu ermöglichen. Je frühzeitiger und intensiver die Rehabilitation startet $(24 \mathrm{~h}$ bis max. 7 Tage nach akutem Ereignis), desto besser scheint das Langzeitergebnis hinsichtlich des Überlebens, der Komplikationen und der Mobilität für diese Patienten zu sein [17, 28, 38]. Jedoch sind für den Erfolg der Frührehabilitation die Dosis der Intervention und die Schwere der neurologischen Schädigung zu berücksichtigen. So profitieren zum Beispiel Patienten mit milder Schädigung nach Schlaganfall von einem frühen und sehr intensiven Training [7, 8]. Frührehabilitation innerhalb der ersten 16 Stunden bei
Hirninfarkt oder bei Hirnblutungen könnte sich bei hoher Dosis jedoch negativ auf den Schweregrad der Behinderung (modified Rankin Scale) auswirken [4, 39]. Eine aktuelle Datenanalyse von 2.104 Patienten konnte sogar höhere Chancen bezüglich der Dauer bis zum Erreichen der Gehfähigkeit und bezüglich der Selbstständigkeit von Patienten mit schwerem Schlaganfall nachweisen, wenn die früheste Trainingseinheit erst nach 24 Stunden mit eher niedrig dosierter Intensität begann und 2 Wochen konstant ohne Steigerung durchgeführt wurde [3]. Eine ältere Studie mit 1.716 Schlaganfallpatienten konnte ähnliche Ergebnisse aufweisen [28]. In der Untersuchung von Musicco 2003 erhöhten intensive Therapiemaßnahmen innerhalb der ersten 2 Wochen nach dem akuten Schlaganfall die Sterberate innerhalb der Rehabilitation. Im Vergleich dazu reduzierte sich die Sterberate bei einem Trainingsstart nach 14 Tagen um das Doppelte. Bei zu langer Verzögerung der Therapiemaßnahmen ( $>30$ Tage nach Akutereignis) 


\section{Evidence based concepts for regaining motor function in early neurological and neurosurgical rehabilitation (ENNR)}

S. Thomas, M. Pohl, J. Mehrholz

\section{Abstract}

Early rehabilitation in patients after stroke is important for regaining functional independence. However, successful rehabilitation depends on the severity of the lesion and therapy intensity. Initiating early progressive physical treatment within the first 24 hours or during the first week in patients with severe stroke results in more clinical complications without increased improvement in motor function compared to controls. However, mildly impaired stroke patients benefit from intensive practice within the first seven days after stroke. No more than two weeks after stroke, training intensity should be increased in both severe and mildly impaired patients. Patients with prolonged mechanical ventilation and intensive care unitacquired weakness (ICUAW) may be mobilized within the first 48 hours after acute critical illness, and intensive mobility treatment may be performed within the first 4 weeks of early rehabilitation without complications.

Motor recovery thus depends on prompt treatment, and also on the training program. Constrained-induced movement therapy is preferred in stroke patients with mild upper limb paresis, whereas severe motor loss can be effectively treated with uni- or bilateral robot-assisted devices. Impaired walking ability after stroke is common and might be reduced with the help of electro-mechanical walking devices, e.g., training with endeffector systems. An increase in walking speed, gait capacity and ability can be achieved by speed-dependent treadmill training in walking stroke patients (FAC $\geq 3$ ). ICUAW syndrome is also common and can be treated by early active mobilization in standing and walking. Other active training methods, e.g., breathing exercises, strength and endurance training can be implemented during early rehabilitation..

Keywords: ENNR, arm function, ambulation

Neurol Rehabil 2016; 22(3): 209-216

(C) Hippocampus Verlag 2016
Schädigung ist weiterhin entscheidend, welche Therapieinhalte angewendet werden. Nachfolgend soll eine Übersicht über mögliche therapeutische Verfahren zur Verbesserung der Motorik bei neurologischen Patienten in der Frührehabilitation gegeben werden.

\section{Schwerpunkt Therapiemaßnahmen}

\section{Motorisches Training der oberen Extremität}

\section{Bilaterales und unilaterales Armtraining}

Bei Patienten nach einem Schlaganfall wird therapeutisch oft debattiert, ob ein bilaterales oder unilaterales Armtraining sinnvoller ist. Das bilaterale Armtraining wird vor allem bei schweren Paresen und dann sehr häufig elektromechanisch assistiert durchgeführt und ermöglicht dem Patienten, mit Hilfe des gesunden Armes den betroffenen Arm während der Bewegung zu unterstützen. Wird unilateral geübt, so wird nur der betroffene Arm ohne die Unterstützung der gesunden Extremität aktiv bewegt, was häufig einen großen Trainingsreiz darstellt. Bei schweren Paresen kann auch das unilaterale Training elektromechanisch assistiert erfolgen (Abb. 1). Häufig wird bei moderaten oder leichten Paresen jedoch darauf verzichtet. Beide Trainingsvarianten (unilateral oder bilateral) haben ihren Vorteil. Aber welche Art zu

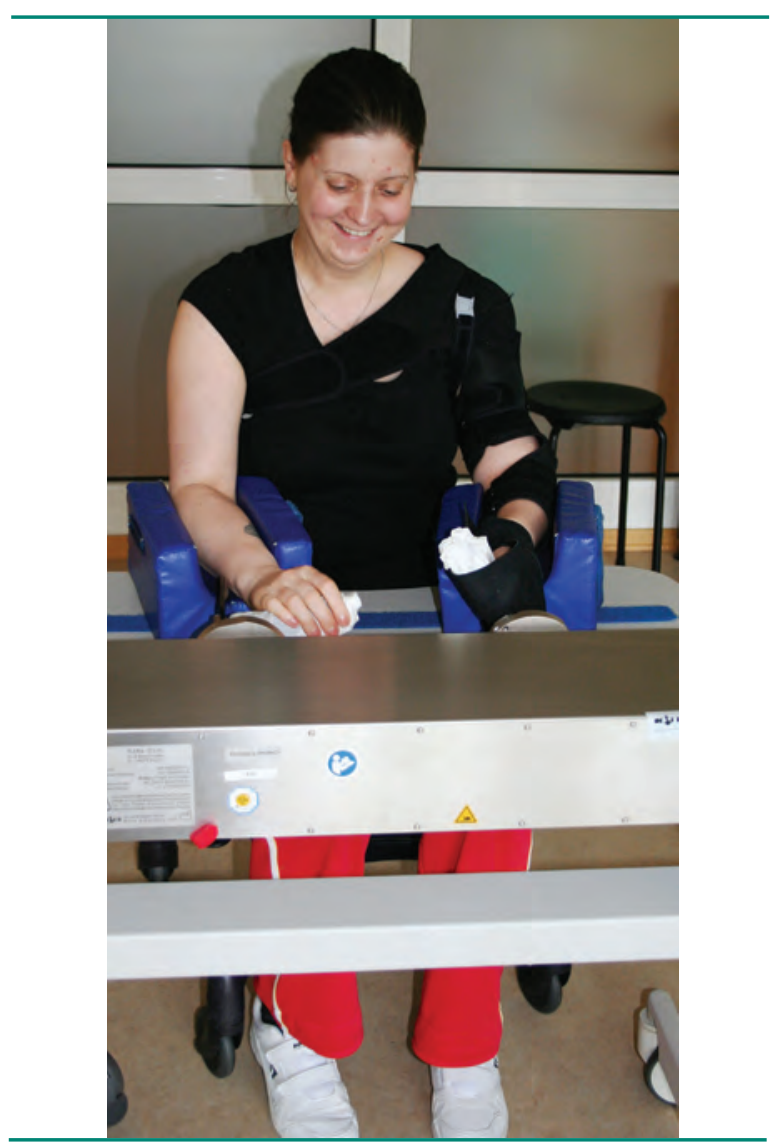

Abb. 1: Bi-Manu-Track 
trainieren ist wann sinnvoll? Eventuell kann das Review von van Delden 2012 eine Antwort geben. Die Autoren untersuchten den Effekt von unilateralem gegenüber bilateralem Training und stratifizierten die Patienten nach der Schwere der Armparese [42]. Sie konnten so einen deutlichen Effekt für das unilaterale Training hinsichtlich des Alltagsgebrauches des Armes (Motor Activity Log) bei milden Paresen feststellen. Bei schweren oder moderaten Paresen gab es laut den Ergebnissen der Studie keinen Unterschied zwischen bilateralem oder unilateralem Training [42]. Bei leichten Armparesen sollte somit das unilaterale Training bevorzugt werden. Hingegen sind bei schweren Armparesen uni- sowie auch bilaterale Trainingsmaßnahmen relativ gleich effektiv.

Patienten mit leichten Armparesen profitieren laut der Studie von van Delden 2012 v. a. von der ConstrainedInduced Movement Therapy (CIMT) [40] hinsichtlich des Armeinsatzes im Alltag [42]. Beim CIMT wird mit einem Handschuh oder einer Armbandage der gesunde Arm immobilisiert. Je länger die Immobilisation des gesunden Armes in den Wachstunden, desto besser. Aber auch kurze Intervalle (30 min am Tag) haben bereits einen Effekt auf die Armmotorik der betroffenen Seite [30]. Dass CIMT auch als Konzept im Gruppentherapieangebot alltagstauglich ist, zeigte eine kürzliche Praxisbeschreibung [44].

Ein systematisches Review von Pollock und Kollegen zur Verbesserung der Armfunktionen nach Schlaganfall konnte hingegen im Überblick aller Reviews zur CIMT nur einen geringen Effekt bezüglich Alltagsaktivitäten aufzeigen [34]. Begründet werden kann dies laut den Autoren durch den Einschluss von Studien, bei denen auch moderat bis schwer betroffene Patienten untersucht wurden [34]. Der größte Effekt der CIMT ist jedoch bei Patienten mit milden Paresen bezüglich der alltäglichen Nutzung zu verzeichnen. Zusätzlich zur Immobilisation der gesunden Seite wird bei CIMT häufig der betroffene Arm repetitiv und aufgabenorientiert (AOT) trainiert. Einen deutlichen Hinweis für die Überlegenheit des AOT der oberen Extremität gegenüber anderen Therapieverfahren bezüglich des motorischen und funktionellen Zugewinns gibt es jedoch derzeit noch nicht [33]. Es ist aber bekannt, dass ein solches Training den motorisch-funktionellen Zustand bei milden Paresen im Vergleich zu keiner Therapie deutlich verbessern kann [31]. Patienten mit milden Paresen sollten daher vor allem durch die Immobilisation des gesunden Armes und Nutzung der betroffenen Seite im Alltag therapiert werden. Zusätzliche repetitive aktive Übungen können diese Therapieform sinnvoll ergänzen.

\section{Frühes roboterassistiertes Training}

Bei Patienten mit schweren Armparesen kann das Training von bilateralen oder unilateralen Armbewegungen mit Hilfe von Robotern effektiv durchgeführt werden [21]. Das sogenannte elektromechanische bzw. roboterassis- tierte Armtraining scheint eine effektive Möglichkeit, um bei Patienten mit schwerer Parese mit hoher Wiederholungszahl zu üben. Eine kürzlich durchgeführte systematische Übersichtsarbeit nach Cochrane Standards schloss insgesamt 34 randomisierte und kontrollierte Studien mit insgesamt 1.061 Patienten ein. Insgesamt zeigte sich, dass elektromechanisch assistierte Armtraining sowohl signifikant die Armkraft, die Armmotorik als auch basale Alltagsfunktionen verbessern hilft [21]. Allerdings sollten Armroboter lediglich als Vehikel gesehen werden, die eine höhere Erholungszahl applizieren helfen und es erlauben, »ohne Ermüdung « mit Patienten sehr häufig (bis zu 1.00o Wiederholung pro Therapiesitzung) zu üben.

Wahrscheinlich ist, dass die Häufigkeit der aufgabenspezifischen Armaktivierung und die stetige aktive Aufmerksamkeit auf den schwer betroffenen Arm, egal mit welcher Methode, eine bedeutende und ausschlaggebende Komponente einer motorischen Therapie ist. Zwar deuten die Ergebnisse des Cochrane Reviews für die Erholung der Armmotorik an, dass eine eher distal ansetzende roboterassistierte Therapie etwas vielversprechender für das Erreichen von Aktivitäten des täglichen Lebens war. Allerdings spielte der Gerätetyp nicht die ausschlaggebende Rolle zum Erreichen von Armfunktionen [21]. Ebenfalls zeigte sich eben ausdrücklich nicht, dass Finger-Hand-Roboter vor allem Finger-HandFunktion verbesserten, und auch Roboter, die vor allem Schulterkomponenten übten, die proximale Armfunktion beeinflussen konnten [21]. Kurz zusammengefasst: Eine Vielzahl an elektromechanisch assistierten Interventionen trägt dazu bei, dass der Patient beim häufigen Üben nicht ermüdet und herausgefordert wird, sodass die Konzentration ständig auf dem schwer betroffenen Arm liegt.

\section{Nicht invasive Hirnstimulation}

Weiterhin ist die Anwendung der nicht invasiven Hirnstimulation für den Erwerb motorischer Fähigkeiten vielversprechend. Methoden wie die repetitive Magnetstimulation (rTMS) und die transkranielle Gleichstromstimulation (tDCS) könnten theoretisch dazu beitragen, die Erholung zu beschleunigen. Jedoch scheint es, als ob die rTMS nur innerhalb der ersten zwei Wochen nach Akutereignis und bei feinmotorischen Störungen bedeutende Effekte erzielen kann [16]. Das Cochrane Review von Hao 2013 zeigt keine eindeutige Evidenz und gibt keine Empfehlung für einen routinemäßigen Einsatz, weder in der neurologischen Rehabilitation noch für die Frührehabilitation, da die meisten Studienergebnisse keinen klaren Effekt aufweisen [15]. Anders könnte es bei der Anwendung von tDCS aussehen. Hier konnten die Autoren einen Nachweis eines allerdings sehr geringen Effektes hinsichtlich der Verbesserung von Alltagsfunktionen ermitteln [10]. Allerdings verbesserten sich Armfunktion durch tDCS nicht [10]. Die tDCS hat ebenfalls keinen Effekt auf die Spastik der oberen Extremitäten [11]. 


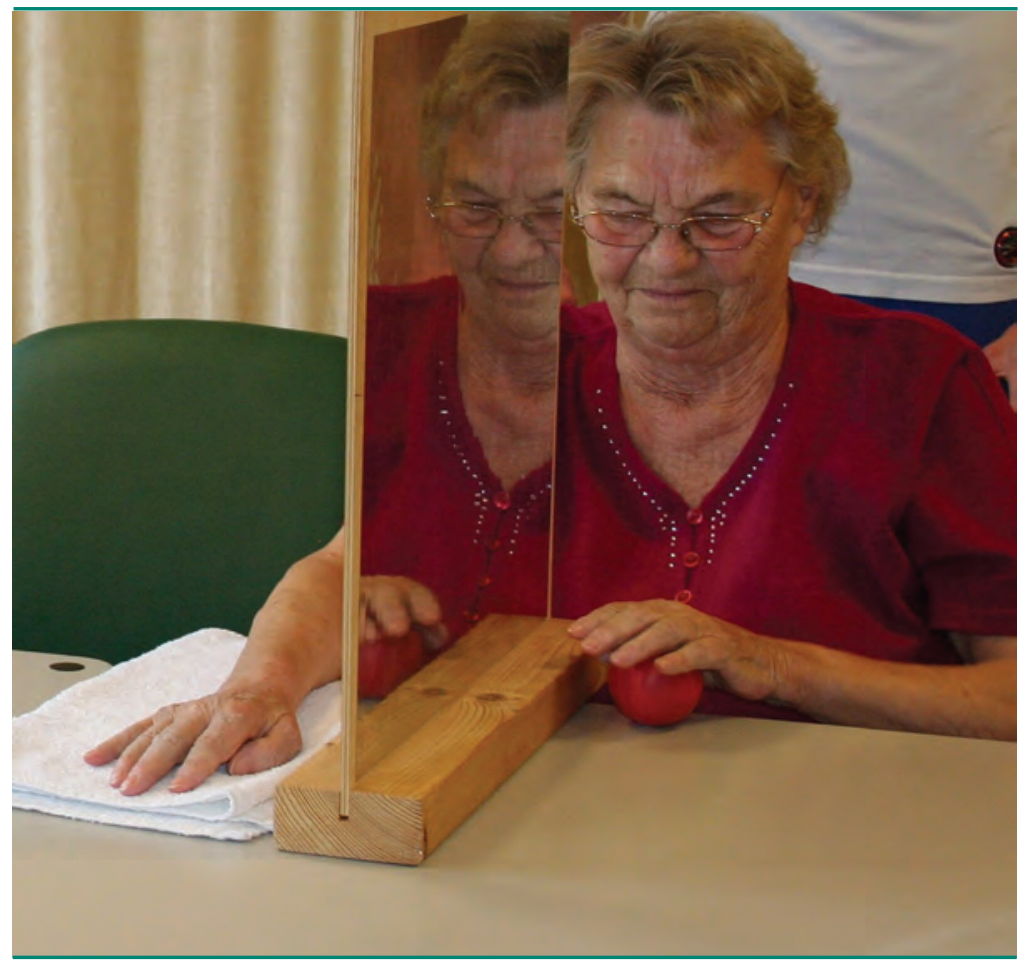

Abb. 2: Spiegeltherapie

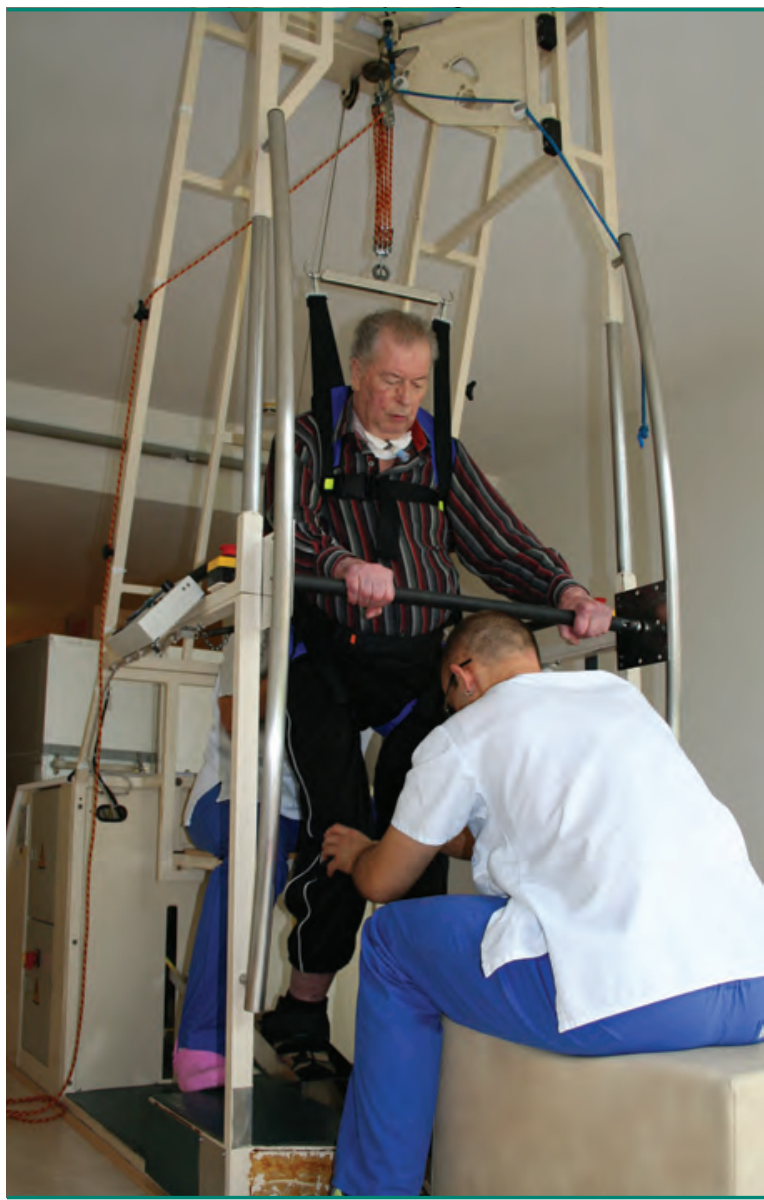

Abb. 3: Gangtrainer

\section{Spiegeltherapie}

Die Spiegeltherapie kann v. a. bei Patienten nach Schlaganfall mit schweren bis moderaten und distalen Armparesen zur Wiederherstellung motorischer Funktionen nach Schlaganfall beitragen [41]. Eine Grundvoraussetzung ist hierbei ein hohes Maß an Konzentrationsfähigkeit, da der Blick des Patienten aktiv im Spiegel gehalten werden muss (Abb. 2). Weiterhin ist eine gute Koordinationsfähigkeit der gesunden Hand erforderlich, die während des Übens im Spiegel beobachtet werden soll. Die Patienten sollten für die Durchführung der Therapie aufrecht im Stuhl sitzen und den Kopf gegen die Schwerkraft halten können. In der frühen neurologischen Rehabilitation stellen diese Ansprüche an den Patienten erfahrungsgemäß eine deutliche Herausforderung dar, sodass häufig auf diese Therapieform verzichtet werden muss. Studien zur Spiegeltherapie existieren bislang auch nur in der chronischen Phase ( $\geq 3$ Monate nach Schlaganfall). Daher ist diese Therapieform in den Phasen C und D eher möglich. Ähnlich verhält es sich auch mit mentalen Trainingsstrategien [2, 5].

\section{Frühes Gehtraining}

\section{Elektromechanische Gangrehabilitation}

Patienten, die nach einem Schlaganfall nicht mehr gehen können, profitieren deutlich von einem frühen Gehtraining. Vor allem mit Hilfe der elektromechanischen Gangtherapie lassen sich Behinderungen im Alltag bei nicht gehfähigen Patienten häufiger vermeiden als mit einem normalen Gehtraining auf der Ebene [22]. Bezüglich der Gehgeschwindigkeit profitieren nicht gehfähige Patienten v. a. von den Endeffektorsystemen (Abb. 3) [22]. Die roboterassistierte Gangtherapie ermöglicht durch die Sicherung der Patienten mit einem Gurtsystem und die mögliche Körpergewichtsentlastung und Schrittführung ein sehr frühes Gehtraining, das nach eigener Erfahrung zeitig nach dem Akutereignis innerhalb der Frührehabilitation angewendet werden kann. Dies bestätigt auch die Patientenpopulation einer Studie von Peurala 2009. Keine andere Studie führte das elektromechanische Gehtraining so zeitig durch [29]. Die Patienten in der Studie von Peurala 2009 erhielten die Therapie bereits innerhalb der 2. Woche nach dem akuten Schlaganfall und konnten die Gehfähigkeit nach dem Training mit dem Gangtrainer im Vergleich zum Gehen auf der Ebene kurzfristig und langfristig deutlich verbessern. Bei näherer Betrachtung der Patienten wird jedoch deutlich, dass die Drop-outRate bei Patienten, die - unabhängig von der Intervention - innerhalb der ersten Woche behandelt wurden, sehr hoch war [29]. Es empfiehlt sich daher, innerhalb der Frührehabilitation ein roboterassistiertes Gehtraining erst in der zweiten postakuten Woche zu beginnen. Außerdem sollte der Trainingsreiz innerhalb der zweiten Woche nicht zu groß sein. Anfangs kann z.B. nur die Dauer der Gangtherapie gesteigert werden. Im weiteren 
Verlauf kann die Schrittgeschwindigkeit erhöht und die Körpergewichtsentlastung verringert werden. Dabei ist es stets wichtig, die Vitalfunktionen zu überprüfen. Das Ziel sollte sein, so viele Schritte wie möglich in der Therapiezeit zu absolvieren. Die roboterassistierte Gangtherapie schließt aber das Üben des Stehens und Gehens in der Ebene nicht aus. Im Gegenteil: Es scheint nach eigener Erfahrung effektiv zu sein, wenn sich beide Therapieformen ergänzen oder im Wechsel durchgeführt werden.

\section{Laufbandtraining}

Patienten nach Schlaganfall, die zumindest 10-15 Meter in Begleitung in der Ebene gehen können (Functional Ambulation Categories [FAC] = 3), profitieren vor allem von einem Laufbandtraining bezüglich Gehausdauer, Gehfähigkeit und Gehgeschwindigkeit [24]. Es bietet sich dabei an, das Training unter Körpergewichtsentlastung mittels eines Gurtsystems durchzuführen [24] (Abb. 4). Um die Belastung des Patienten während des Trainings zu steuern, empfiehlt es sich, bei einem Ausdauertraining die optimale Herzfrequenz mittels der KarvonenFormel zu berechnen [20].

\section{ohne Betablocker:}

untere Belastungsgrenze $=$

([(220-Alter) - Ruhepuls $]$ * 40\%) + Ruhepuls

obere Belastungsgrenze $=$

([(220-Alter) - Ruhepuls $]$ * 85\%)+ Ruhepuls

\section{mit Betablockern:}

untere Belastungsgrenze $=$

$\left[([(220-\text { Alter }) \text { * } 0,85]-\text { Ruhepuls })^{\star} \quad 40 \%\right]+$ Ruhepuls obere Belastungsgrenze $=$

$\left[\left(\left[(220-\right.\right.\right.$ Alter $){ }^{\star}$ o,85]-Ruhepuls $)$ * $\left.85 \%\right]+$ Ruhepuls

Gehgeschwindigkeit, Schrittlänge sowie Gehfähigkeit von chronischen Schlaganfallpatienten ( $\geq 3$ Monate, mind. $\mathrm{FAC}=3$ ) können durch ein schnelles und intensives Gehtraining auf dem Laufband verbessert werden [32]. Aber auch gehfähige Patienten (mind. $\mathrm{FAC}=3$ ) innerhalb der neurologischen Frührehabilitation profitieren hinsichtlich der Gehgeschwindigkeit und Schrittlänge von dem frühzeitigen Sprinttraining (12 Tage nach dem Akutereignis) [18]. In beiden Untersuchungen wurde das Laufbandtraining ähnlich absolviert:

- Start: Gehen auf dem Laufband bei maximal möglicher Gehgeschwindigkeit

- Steigerung der Gehgeschwindigkeit um jeweils 10\% für 10-30 s (jeweils Erholungspausen zwischen den Einheiten

- bei Stolpern Gehgeschwindigkeit um 10\% senken

- maximal 5 Erhöhungen der Gehgeschwindigkeit pro Trainingstag $[18,32]$

Beide Studien konnten einen deutlichen Vorteil für das Sprinttraining auf dem Laufband im Vergleich zum

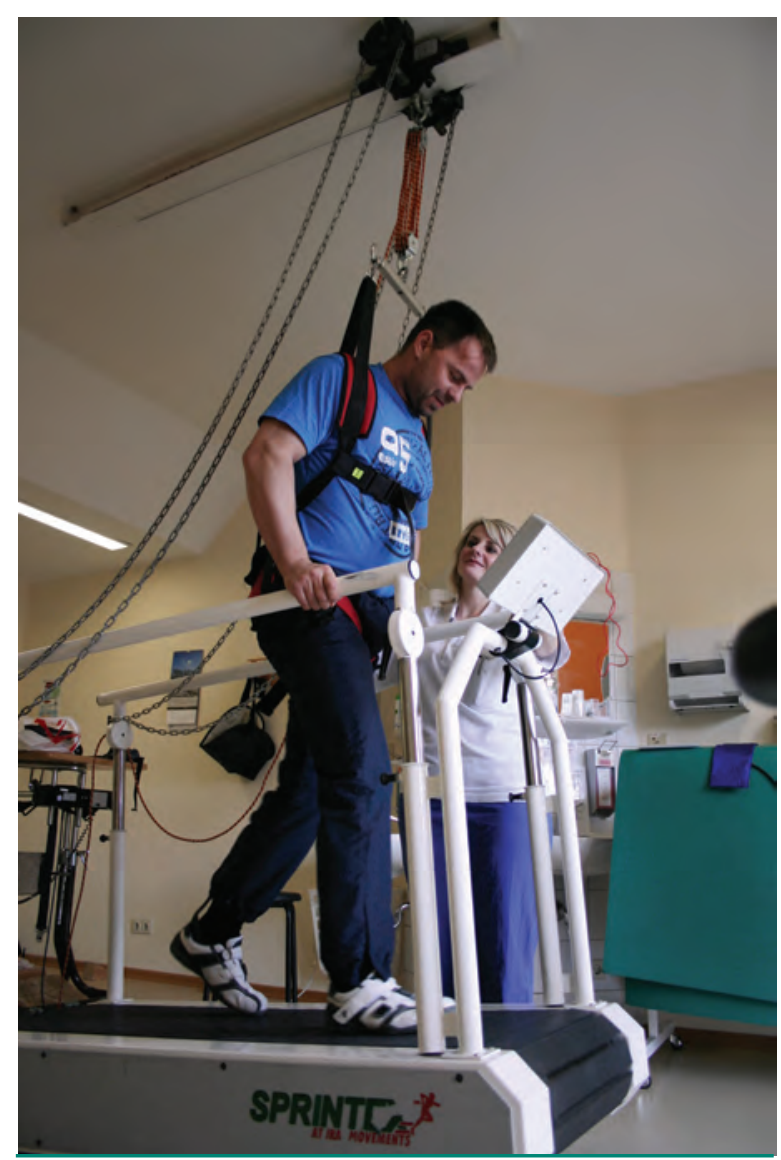

Abb. 4: Laufband mit Gurtsystem

Laufbandtraining mit komfortabler Gehgeschwindigkeit nachweisen. Schrittlänge, Gehgeschwindigkeit und Gehfähigkeit konnte bei Patienten, die mindestens in Begleitung in der Ebene gehen konnten, gesteigert werden. Bezüglich Patienten, die nicht gehfähig sind, daher körperliche Hilfestellung von Hilfspersonen benötigen, ist das Laufbandtraining hinsichtlich der erwähnten Parameter im Vergleich zu anderen Therapien nicht überlegen [24]. Bei diesen schwerbetroffenen Patienten sollte an die elektromechanische Gangtherapie gedacht werden.

\section{Frührehabilitation bei bestehender Intensivbehandlungspflicht}

Die meisten Intensivpatienten, die die Akutphase überleben, können häufig frühzeitig von intensivmedizinischen Maßnahmen wie der maschinellen Beatmung entwöhnt werden (Weaning). Es gibt aber auch Patienten, bei denen das Weaning nur sehr langsam voranschreitet oder gar stagniert. Diese Patienten weisen eine deutliche Schwäche der Atemmuskulatur und der Arme und Beine unter anderem aufgrund einer Critical-Illness-Polyneuropathie und/oder -myopathie, auf. Um Alltagsbewegungen wie Stehen und Gehen für diese Patienten wieder zu ermöglichen, müssen diese früh intensiv rehabilitiert 


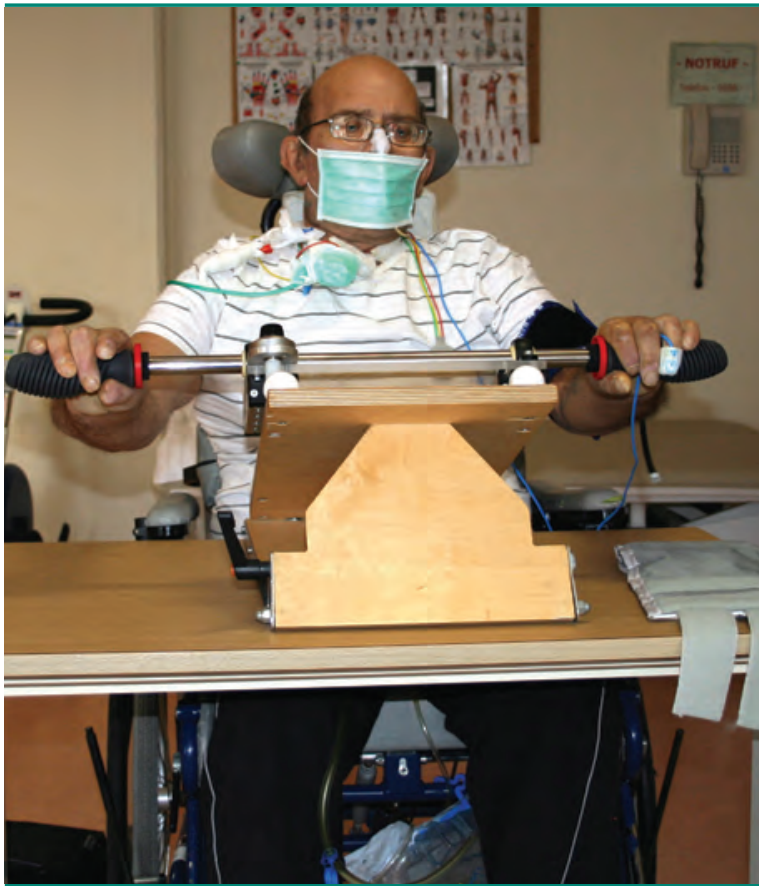

Abb. 5: Armtraining

werden. Eine Herausforderung in der Behandlung dieser Patienten mit dem »auf der ITS-erworbenen Schwächesyndrom « besteht jedoch in der oftmals ausgeprägten Komorbidität dieser Patienten. Trotzdem sind, wie auch in der Frührehabilitation von Patienten mit erworbener Hirnschädigung, aktive Maßnahmen der therapeutische Hauptschwerpunkt. Zu nennen sind v. a. die allgemeine Mobilisierung in den Sitz, das Training des selbstständigen Aufstehens, des Stehens und Gehens [27]. Die frühzeitige Mobilisierung dieser speziellen Patienten (24-48 Stunden nach Aufnahme) wird, nach Stabilisierung der Hämodynamik, in der Literatur eindeutig empfohlen [1, 6, 13]. Bei der Mobilisierung inaktiver Patienten wird als Minimum empfohlen, 3-mal täglich je 20 Minuten im Bett zu sitzen [1]. Sobald ein Arm aktiv gegen die Schwerkraft gehoben werden kann, sollte das selbstständige Sitzen an der Bettkante für mindestens 20 Minuten/ Tag geübt werden [1]. Bei möglichen Beinbewegungen gegen die Schwerkraft kann der Patienten mindestens 20 Minuten/Tag in einem Stuhl sitzen [26]. Ein zusätzliches Armtraining kann dabei zur Belastungssteigerung und zur Minderung von Dyspnoe beitragen [35] (Abb. 5). Im Sitz können zusätzliche atemtherapeutische Übungen die Lungenperfusion verbessern und die erhöhte Herzfrequenz senken [9, 36]. Zur Verbesserung der Muskelkraft der unteren Extremität und der Ausdauer kann bereits schon in Rückenlage das Training mit einem adaptierten Liegeergometern kontinuierliche durchgeführt werden [6]. Ein aktives Training der inspiratorischen Atemmuskulatur kann die Dauer der Beatmung deutlich reduzieren [19]. Passive Bewegungsübungen gerade bei sedierten Patienten oder schwersten Paresen kann den Muskelumbau bei beatmeten Patienten positiv beeinflussen [14]. Auch elektrotherapeutische Verfahren zur Aktivierung der Muskulatur sind bei sedierten oder vollständig immobilen Patienten einsetzbar, um das Ausmaß der Atrophie zu reduzieren [12, 37, 43]. Die Entwicklung dieser Patienten hinsichtlich motorischer Fähigkeiten unter Therapiemaßnahmen ist bislang jedoch kaum evaluiert. Eine aktuelle Kohortenstudie konnte bei diesen Patienten unter täglicher frühzeitiger Physiotherapie eine deutliche Verbesserung der Gehfähigkeit, Kraft, Ausdauer und Balance innerhalb der ersten vier Wochen feststellen [23]. Es existieren bislang jedoch keine randomisierten kontrollierten Studien zu Therapieverfahren bei chronisch kritisch kranken Patienten mit erworbenem Schwächesyndrom, durch welche eine klare Empfehlung zu bestimmten frühzeitigen Therapieverfahren gegeben werden kann [25].

\section{Schlussfolgerung}

Die motorische Rehabilitation von Patienten mit erworbener Hirnschädigung sollte so früh wie möglich abhängig vom Schweregrad der Störung starten. Patienten mit leichten Paresen profitieren von einem intensiven Training der Armmotorik und Gehfähigkeit schon innerhalb der ersten $24 \mathrm{~h}$ bis 7 Tage nach dem Akutereignis. Patienten mit schwerer Schädigung sollten innerhalb der ersten Woche mit sehr niedriger Intensität therapiert werden. Innerhalb derzweiten Woche kann das Training bei schwerer Schädigung langsam gesteigert und nach 14 Tagen intensiviert werden. Um die Armmotorik in der Frührehabilitation zu verbessern, eignet sich bei leichten Paresen v. a. die Constrained Induced Movement Therapy. Patienten mit schweren Paresen können v. a. von einem robotergestützten bi- oder unilateralen Training profitieren. Die Gehfähigkeit kann mit Hilfe des elektromechanischen Gangtrainings bei nicht gehfähigen Patienten innerhalb der zweiten Woche nach dem Akutereignis erfolgen. Gehfähige Patienten (mind. FAC $=3$ ) können mit einem gezielten Laufbandtraining Gehgeschwindigkeit, Gehfähigkeit $(\mathrm{FAC}=4 / 5)$ und Gehausdauer verbessern. Bei Patienten mit andauernder kritischer Situation und prolongiertem Weaning können im Rahmen der Frührehabilitation vor allem in den ersten vier Wochen aktive, mobilisierende Therapiemaßnahmen mit hoher Intensität erfolgen.

\section{Abkürzungen}
AOT aufgabenorientiertes Training
BMT bimanuelles Training
CIMT Contrained Induced Movement Therapy
FAC Functional Ambulation Categories
ITS Intensivstation
rTMS repetitive Magnetstimulation
tDCS transkranielle Gleichstromstimulation 


\section{Literatur}

1. Bailey P, Thomsen GE, Spuhler VJ, Blair R, Jewkes J, Bezdjian L, Veale K, Rodriquez L, Hopkins RO. Early activity is feasible and safe in respiratory failure patients. Crit Care Med 2007; 35: 139-145.

2. Barclay-Goddard RE, Stevenson TJ, Poluha W, Thalman L. Mental practice for treating upper extremity deficits in individuals with hemiparesis after stroke. Cochrane Database Syst Rev 2011; CDo05950.

3. Bernhardt J, Churilov L, Ellery F, Collier J, Chamberlain J, Langhorne P, Lindley R, Moodie M, Dewey H, Thrift A, Donnan G. Prespecified dose-response analysis for A Very Early Rehabilitation Trial (AVERT). Neurol 2016; DOI 10.1212/WNL.0000000000002459.

4. Bernhardt J, Langhorne P, Lindley RI, Thrift AG, Ellery F, Collier J, Churilov L, Moodie M, Dewey H, Donnan G. Efficacy and safety of very early mobilisation within 24 $\mathrm{h}$ of stroke onset (AVERT): a randomised controlled trial. Lancet 2015; 386: 46-55.

5. Braun S, Kleynen M, van Heel T, Kruithof N, Wade D, Beurskens A. The effects of mental practice in neurological rehabilitation; a systematic review and meta-analysis. Front Hum Neurosci 2013; 7: 390.

6. Burtin C, Clerckx B, Robbeets C, Ferdinande P, Langer D, Troosters T, Hermans G, Decramer M, Gosselink R. Early exercise in critically ill patients enhances short-term functional recovery. Crit Care Med 2009; 37: 2499-2505.

7. Craig LE, Bernhardt J, Langhorne P, Wu O. Early mobilization after stroke: an example of an individual patient data meta-analysis of a complex intervention. Stroke 2010; 41: 2632-2636.

8. Cumming T, Thrift A, Collier J, Churilov L, Dewey H, Donnan G, Bernhardt J. Very Early Mobilization After Stroke Fast-Tracks Return to Walking: Further Results From the Phase II AVERT Randomized Controlled Trial. Stroke 2011; 42: $153-158$.

9. Dean E. Effect of body position on pulmonary function Phys Ther 1985; 65: 613-618.

10. Elsner B, Kugler J, Pohl M, Mehrholz J. Transcranial direct current stimulation (tDCS) for improving activities of daily living, and physical and cognitive functioning, in people after stroke. Cochrane Database Syst Rev 2016; 3 : CDoog645.

11. Elsner B, Kugler J, Pohl M, Mehrholz J. Transcranial Direct Current Stimulation for improving spasticity after stroke. A systematic review with meta-analysis. Journal of Rehabilitation Medicine 2016; accepted 07-03-2016 - In Publication - Copy Editing.

12. Gerovasili V, Stefanidis K, Vitzilaios K, Karatzanos E, Politis P, Koroneos A, Chatzimichail A, Routsi C, Roussos C, Nanas S. Electrical muscle stimulation preserves the muscle mass of critically ill patients: a randomized study. Crit Care 2009; 13: R161.

13. Gosselink R, Bott J, Johnson M, Dean E, Nava S, Norrenberg M, Schonhofer B, Stiller K, van de Leur H, Vincent JL. Physiotherapy for adult patients with critical illness: recommendations of the European Respiratory Society and European Society of Intensive Care Medicine Task Force on Physiotherapy for Critically Ill Patients. Intensive Care Med 2008; 34: 1188-1199.

14. Griffiths RD, Palmer TE, Helliwell T, MacLennan P, MacMillan RR. Effect of passive stretching on the wasting of muscle in the critically ill. Nutrition 1995; 11: 428-432.

15. Hao Z, Wang D, Zeng Y, Liu M. Repetitive transcranial magnetic stimulation for improving function after stroke. Cochrane Database Syst Rev 2013; 5: CDoo8862.

16. Khedr EM, Abdel-Fadeil MR, Farghali A, Qaid M. Role of 1 and $3 \mathrm{~Hz}$ repetitive transcranial magnetic stimulation on motor function recovery after acute ischaemic stroke. Eur J
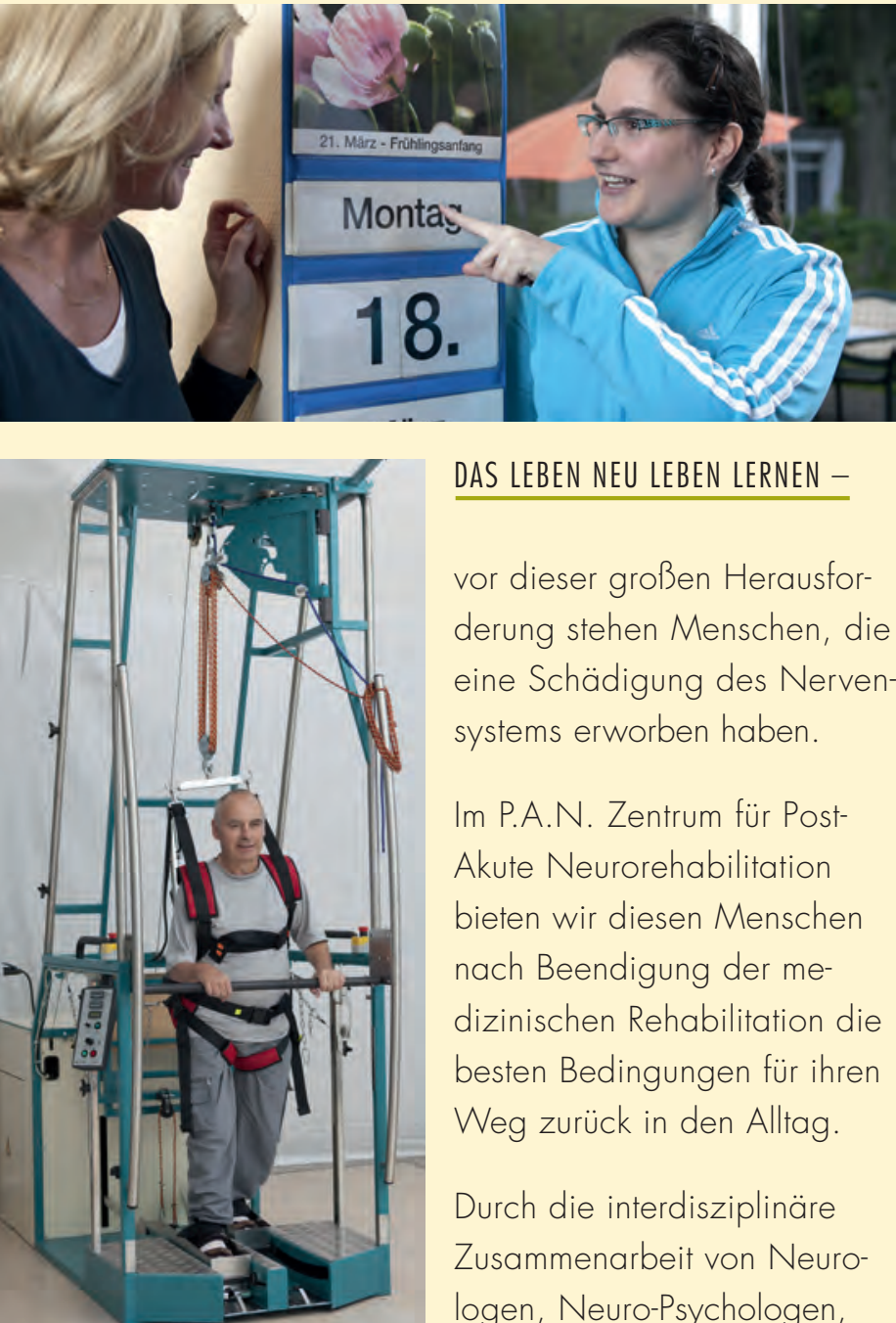

DAS LEBEN NEU LEBEN LERNEN -

vor dieser großen Herausforderung stehen Menschen, die eine Schädigung des Nervensystems erworben haben.

Im P.A.N. Zentrum für PostAkute Neurorehabilitation bieten wir diesen Menschen nach Beendigung der medizinischen Rehabilitation die besten Bedingungen für ihren Weg zurück in den Alltag.

Durch die interdisziplinäre Zusammenarbeit von Neurologen, Neuro-Psychologen, Neuro-Pädagogen und

Therapeuten, sowie durch die Einbeziehung aktueller Forschungsergebnisse erkennen wir das Potenzial jedes Einzelnen und schöpfen es aus.

Unser Ziel ist es, die Menschen wieder zu befähigen, ambulant und möglichst selbstständig zu leben. Vielen gelingt dieser Schritt innerhalb von 18 bis 24 Monaten.

P.A.N. ZENTRUM FÜR POST-AKUTE NEUROREHABILITATION IM FÜRST DONNERSMARCK-HAUS BERLIN-FROHNAU EINE EINRICHTUNG DER FÜRST DONNERSMARCK-STIFTUNG

Raventaler Str. 32 | 13465 Berlin

Es berät Sie: Prof. Dr. med. Stephan Bamborschke

Leitender Arzł des P.A.N. Zentrums

Tel. (030) 40 606-231 I Fax (030) $40606-340$

E-Mail: bamborschke.fdh@fdst.de

www. panzentrum.de 
Neurol 2009; 16: 1323-1330.

17. Langhorne P, Stott D, Knight A, Bernhardt J, Barer D, Watkins C. Very Early Rehabilitation or Intensive Telemetry after Stroke: A Pilot Randomised Trial. Cerebrovasc Dis 2009; 29: 352-360.

18. Lau KW, Mak MK. Speed-dependent treadmill training is effective to improve gait and balance performance in patients with sub-acute stroke. J Rehabil Med 2011; 43: 709-713.

19. Martin AD, Smith BK, Davenport PD, Harman E, GonzalezRothi RJ, Baz M, Layon AJ, Banner MJ, Caruso LJ, Deoghare $\mathrm{H}$, Huang TT, Gabrielli A. Inspiratory muscle strength training improves weaning outcome in failure to wean patients: a randomized trial. Crit Care 2011; 15: R84.

20. Mehrholz J. Ausdauertraining nach Schlaganfall. Pages 123-134 In: J. Mehrholz, ed. Neuroreha nach Schlaganfall. Georg Thieme Verlag Stuttgart 2011, 123-134.

21. Mehrholz J. Internationale Studienergebnisse. neuroreha 2015; 07: 148-150.

22. Mehrholz J, Elsner B, Werner C, Kugler J, Pohl M.Electromechanical-assisted training for walking after stroke. Cochrane Database Syst Rev 2013; 7: CDoo6185

23. Mehrholz J, Muckel S, Oehmichen F, Pohl M. First results about recovery of walking function in patients with intensive care unit-acquired muscle weakness from the General Weakness Syndrome Therapy (GymNAST) cohort study. BMJ Open 2015; 5: eoo8828.

24. Mehrholz J, Pohl M, Elsner B. Treadmill training and body weight support for walking after stroke. Cochrane Database Syst Rev 2014; 1: CDoo2840.

25. Mehrholz J, Pohl M, Kugler J, Burridge J, Muckel S, Elsner B. Physical rehabilitation for critical illness myopathy and neuropathy. Cochrane Database Syst Rev 2015; 3: CDo10942.

26. Morris PE, Griffin L, Berry M, Thompson C, Hite RD, Winkelman C, Hopkins RO, Ross A, Dixon L, Leach S, Haponik E. Receiving early mobility during an intensive care unit admission is a predictor of improved outcomes in acute respiratory failure. Am J Med Sci 2011; 341: 373-377.

27. Mückel S, Mehrholz K, Mehrholz J, Pohl M. Behandlungsansätze bei Patienten mit auf der Intensivstation erworbenen Schwächesyndrom. neuroreha 2013; 13: 40-43.

28. Musicco M, Emberti L, Nappi G, Caltagirone C. Early and Long-Term Outcome of Rehabilitation in Stroke Patients: The Role of Patient Characteristics, Time of Initiation, and Duration of Interventions. Arch Phys Med Rehabil 2003; 48: $551-558$

29. Peurala SH, Airaksinen O, Huuskonen P, Jakala P, Juhakoski M, Sandell K, Tarkka IM, Sivenius J. Effects of intensive therapy using gait trainer or floor walking exercises early after stroke. J Rehabil Med 2009; 41: 166-173.

30. Peurala SH, Kantanen MP, Sjogren T, Paltamaa J, Karhula M, Heinonen A. Effectiveness of constraint-induced movement therapy on activity and participation after stroke: a systematic review and meta-analysis of randomized controlled trials. Clin Rehabil 2012; 26: 209-223.

31. Platz T, Winter T, Muller N, Pinkowski C, Eickhof C, Mauritz KH. Arm ability training for stroke and traumatic brain injury patients with mild arm paresis: a single-blind, randomized, controlled trial. Arch Phys Med Rehabil 2001; 82: 961-968.

32. Pohl M, Mehrholz J, Ritschel C, Ruckriem S. Speed-dependent treadmill training in ambulatory hemiparetic stroke patients: a randomized controlled trial. Stroke 2002; 33: 553-558.

33. Pollock A, Baer G, Campbell P, Choo PL, Forster A, Morris J, Pomeroy VM, Langhorne P. Physical rehabilitation approaches for the recovery of function and mobility following stroke. Cochrane Database Syst Rev 2014; 4: CDoo1920.
34. Pollock A, Farmer SE, Brady MC, Langhorne P, Mead GE, Mehrholz J, van Wijck F. Interventions for improving upper limb function after stroke. Cochrane Database Syst Rev 2014; 11: CD010820.

35. Porta R, Vitacca M, Gile LS, Clini E, Bianchi L, Zanotti E, Ambrosino N. Supported arm training in patients recently weaned from mechanical ventilation. Chest 2005; 128: 2511-2520.

36. Porter JM, Pidgeon C, Cunningham AJ. The sitting position in neurosurgery: a critical appraisal. Br J Anaesth 1999; 82: $117-128$.

37. Routsi C, Gerovasili V, Vasileiadis I, Karatzanos E, Pitsolis T, Tripodaki E, Markaki V, Zervakis D, Nanas S. Electrical muscle stimulation prevents critical illness polyneuromyopathy: a randomized parallel intervention trial. Crit Care 2010; 14: R74.

38. Sorbello D, Dewey H, Churilov L, Thrift A, Collier J, Donnan G, Bernhardt J. Very Early Mobilisation and Complications in the First 3 Months after Stroke:Further Results from Phase II of A Very Early Rehabilitation Trial (AVERT). Cerebrovasc Dis 2009; 28: 378-383.

39. Sundseth A, Thommessen B, Ronning OM. Outcome after mobilization within 24 hours of acute stroke: a randomized controlled trial. Stroke 2012; 43: 2389-2394.

40. Taub E, Miller NE, Novack TA, Cook EW, 3rd, Fleming WC, Nepomuceno CS, Connell JS, Crago JE. Technique to improve chronic motor deficit after stroke. Arch Phys Med Rehabil 1993; 74: 347-354.

41. Thieme H, Mehrholz J, Pohl M, Behrens J, Dohle C. Mirror therapy for improving motor function after stroke. Cochrane Database Syst Rev 2013; 3: CDoo8449.

42. van Delden AE, Peper CE, Beek PJ, Kwakkel G: Unilateral versus bilateral upper limb exercise therapy after stroke: a systematic review. J Rehabil Med 2012; 44: 106-117.

43. Zanotti E, Felicetti G, Maini M, Fracchia C. Peripheral muscle strength training in bed-bound patients with COPD receiving mechanical ventilation: effect of electrical stimulation. Chest 2003; 124: 292-296.

44. Zarski C, Rollnik JD. Constraint-Induced Movement Therapy als Gruppentherapie (CIMT) eine Methode wird alltagstauglich. physiopraxis 2010; 8: 30-33.

\section{Interessenvermerk}

Die Autoren geben an, dass kein Interessenkonflikt vorliegt.

\section{Korrespondenzadresse:}

Prof. Dr. rer. medic. habil. Jan Mehrholz

Leiter Wissenschaftliches Institut

Private Europäische Medizinische Akademie der

Klinik Bavaria in Kreischa $\mathrm{GmbH}$

An der Wolfsschlucht 1-2

01731 Kreischa

jan.mehrholz@klinik-bavaria.de 